\title{
Modern Trends in the Development of EPR/ESR
}

\author{
Elena Bagryanskaya ${ }^{1} \cdot$ Hong-In Lee ${ }^{2} \cdot$ \\ Czeslaw Rudowicz ${ }^{3} \cdot$ Hitoshi Ohta ${ }^{4}$
}

This issue of Applied Magnetic Resonance is devoted to show modern trends in the development of electron paramagnetic resonance/electron spin resonance (EPR/ ESR). EPR/ESR researches have expanded from researches in physics and chemistry to those in biology, medicine or materials science. For the development of EPR/ESR method, one of recent trends is to expand into high-frequency and high-field regions together with multi-frequencies.

Although the papers collected in this issue cannot cover all aspects of modern trends in the development and the application of EPR/ESR, they may give a flavor on the current state of the art of EPR/ESR research. Let us survey in short about the content of the papers in this interesting issue.

Multi-frequency ESR studies were shown by many authors. T. Sakurai, R. Matsui, K. Kawasaki, S. Okubo, H. Ohta, K. Matsubayashi, Y. Uwatoko, K. Kudo and Y. Koike described their development of high-pressure and multi-frequency ESR system. K. Kawasaki, T. Sakurai, E. Ohmichi, S. Okubo, H. Ohta, K. Matsubayashi and Y. Uwatoko also described their new development of highpressure ESR system using the micro-coil. Four papers written by D. Yoshizawa, T. Kida, S. Nakatsuji, K. Iritani, M. Halim, T. Takeuchi and M. Hagiwara, A. Okutani, S. Kimura, T. Takeuchi and M. Hagiwara, S. Kimura, K. Watanabe, T. Kashiwagi, H. Yamaguchi, M. Hagiwara and Z. Honda, S. Ikeda, S. Hara, T. Sakurai, S. Okubo, H. Ohta and H. Sakurai showed multi-frequency ESR studies of various magnetic

Hitoshi Ohta

hohta@kobe-u.ac.jp

$1 \quad$ Novosibirsk Institute of Organic Chemistry, 630090 Novosibirsk, Russia

2 Department of Chemistry, Kyungpook National University, Daegu 702-701, Republic of Korea

3 Institute of Physics, West Pomeranian University of Technology Szczecin, 70-310 Szczecin, Poland

4 Molecular Photoscience Research Center, Kobe University, Kobe 657-8501, Japan 
systems using high magnetic field. T. Sakai, K. Hijii, S. Okubo, H. Ohta, H. Nakano and S. Miyashita discussed about the ESR theory on the singlet and triplet transition in Kagome-lattice antiferromagnet.

Several applications using the conventional ESR were also shown. L. R. Goveas, K. S. Bhagyashree, K. N. Anuradha and S. V. Bhat, D. Paul, K. N. Anuradha, K. $\mathrm{S}$ Bhagyashree and S. V. Bhat discussed about the electron magnetic resonance (EMR) studies on manganite. S. M. Kaczmarek, H. Fuks, M. Berkowski, M. Głowacki and B. Bojanowski applied to the study of $\mathrm{NdVO}_{4}$. A. Nakajima, T. Yamaguchi, T. Yamashita, K. Kawai, Y. Miyake, K. Kanaori, K. Tajima discussed their development of a flow-injection spin-trapping ESR system and its application. Finally S. C. Drew, T. Ando and Y. Yonamoto discussed the biological applications.

We hope that the publications presented in this issue will stimulate further progress in EPR/ESR and its applications. 